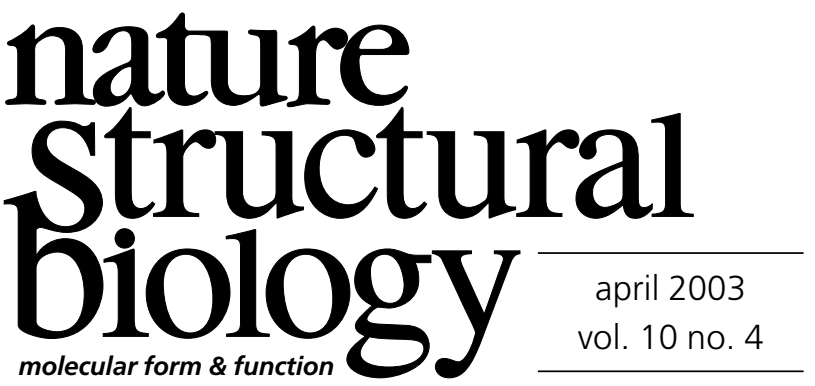

\title{
Painkillers and prostaglandins
}

Prostaglandins are powerful signaling agents in the human body. The two-dozen or so members of this family of small lipid messengers underpin many profound physiological events including vasodilation, vasoconstriction, bronchoconstriction, platelet activation, inflammation, uterine contractions, pain perception and fever. Drugs to counter their less desirable effects have become permanent residents in medicine cabinets around the world, and for a good reason - they are effective, cheap and reasonably safe. Nevertheless, history has shown that, with new information from basic research, a drug can usually be improved. For example, the results of a study reported on page 291 of this issue of Nature Structural Biology shed light on a particular enzyme involved in prostaglandin metabolism. Information such as this may eventually lead to even better prostaglandin-blocking compounds than those available today.

First discovered in seminal fluid in the early 1930s, prostaglandins are now known to be important regulators in many parts of the body. In 1982, the Nobel Prize in Physiology or Medicine was presented to three scientists, Sune K. Bergström, Bengt I. Samuelsson and John R. Vane, for their work on this family of hormones. Vane's contributions include showing that one of the world's most widely used drugs - aspirin — acts to relieve pain, fever and inflammation by blocking the production of prostaglandins. The long history of aspirin and other pain relievers emphasizes the importance of this discovery.

The ancient Egyptians and Romans were already aware that certain plants could be used to treat pain and fever. Hippocrates also championed the chewing of willow bark for pain relief. In England in 1763, the Reverend Edward Stone published a widely read paper promoting the benefits of willow bark extract, and in the 1820 s salicylic acid was identified as the bark's active component. In 1860, a process for mass-producing salicylic acid was discovered. Soon after, many unfortunate patients learned the hard way that the pure form of salicylic acid is not well tolerated, causing diarrhea and vomiting. A search for alternatives ensued, and in the late 1890s, Felix Hoffman of the Bayer Company in Germany found that acetylsalicylic acid was a better choice. It was marketed under the trade name Aspirin, with 'a' for the acetyl group and 'spir' for the botanical genus spiraea, from which salicylates can be extracted.

Aspirin was the main treatment for pain and fever for nearly 60 years. However, its widespread use highlighted significant problems - it could cause peptic ulcers and was linked to Reye's syndrome, a rare but serious problem in children. Once again, alternatives to combat pain and fever were sought.
Thus began the world's current preference for acetaminophen, ibuprofen and other related drugs.

Acetaminophen was identified as a pain reliever in the 1890s, even before aspirin was discovered, but it did not enter widespread use until the 1950s. A small drug company called McNeil Laboratories in Pennsylvania began marketing acetaminophen under the brand name Tylenol in 1955. Unlike aspirin, acetaminophen is strictly an analgesic and antipyretic. It does not reduce inflammation, but nor does it upset the stomach. However, acetaminophen at high doses can cause liver failure. Clearly, no drug is perfect.

In the 1960s, scientists at the Boots Pure Drug Company in the UK discovered that the phenylalkanoic class of acids, of which ibuprofen is a member, are also good pain killers, fever reducers and anti-inflammatory agents. Clinical trials with ibuprofen were conducted in the 1970s, and in the early 1980s it reached drugstore shelves under the trade names of Nuprin, Advil and Motrin. Related compounds, such as naproxen (known as Aleve) and ketoprofen (known as Orudis) are also now available. All of these can cause stomach upset, but usually less so than aspirin.

It is now known that all of these drugs block the activities of cyclooxygenase (COX), enzymes that act early in the pathways of prostaglandin synthesis. So far, three COX enyzymes have been identified: COX-1 is involved in protecting the gastrointestinal tract from acid assault; COX-2 is involved in pain, fever, and inflammation; and the role of the newly discovered COX-3, which is expressed primarily in the brain and heart, remains obscure. Both aspirin and ibuprofen inhibit COX-1 and COX-2, but acetaminophen appears to inhibit only COX-3. Very recently, additional anti-inflammatory agents have been introduced, ones that inhibit COX-2 but not COX-1. These compounds, Celecoxib (known as Celebrex) and rofecoxib (known as Vioxx), have become some of the fastest selling drugs on the market. However, they too have their problems, as they promote blood clotting which may lead to heart attack and stroke in rare cases.

The information from basic research has laid the foundation for the development of drugs against each of the cyclooxygenase subtypes. However, with each powerful drug, new side effects have also emerged. This points to the need to better understand the production and function of various prostaglandins in the body. With increased knowledge in this area, perhaps it will eventually be possible to make yet more specific drugs tailored to each prostaglandin action. One day we may use one drug to dull pain, another to reduce blood clotting, yet another to lower fever, and so forth - a pill for every prostaglandin's purpose. 